\title{
Health-related Quality of Life and Quality of Care in pregnant and postnatal women during the COVID-19 pandemic: a case control study
}

\author{
Fátimah Alaya ${ }^{1}$, Amy P Worrall ${ }^{2}$, Fiona O'Toole ${ }^{2}$, Jillian Doyle ${ }^{2}$, Richard Duffy ${ }^{2}$, and \\ Michael Geary ${ }^{2}$ \\ ${ }^{1}$ Royal College of Surgeons in Ireland \\ ${ }^{2}$ Rotunda Hospital
}

September 28, 2020

\begin{abstract}
Objective To compare self-reported health related quality of life (HRQoL) and quality of care (QoC) received between pregnant and postnatal women during the COVID-19 pandemic at a tertiary unit. Design A prospective case-control study Population Pregnant women, [?]18 years of age, with capacity, who spoke English, and attended the tertiary unit during the pandemic. Methods 18 perinatal women who tested positive for SARS-CoV-2 and 20 asymptomatic control perinatal women were recruited. Demographic characteristics were collected and all participants completed the Short Form (SF-12), Clinical Outcomes in Routine Evaluation-Outcome Measure (CORE-OM) and Quality from the Patient's Perspective (QPP). Means scores in both cohorts were compared. Main Outcome Measures Assessment of psychological distress, HRQoL, QoC and their correlates. Results $95 \%$ of the Non-COVID cohort were Caucasian, while $67 \%$ of the pregnant women who had COVID-19 were not Caucasian $(\mathrm{x} 2=16.01, \mathrm{p}<0.0001)$. The mean SF-12 for physical health and functionality in the COVID cohort had significantly lower scores (36.54 vs 49.21, 95\% CI [6.9 to 20.2], $\mathrm{p}<0.0002$ ). There was no difference in mental health and wellbeing scores between the COVID and Non-COVID cohorts. The QoC experienced by both cohorts was similar and very positive. Conclusions Among pregnant women who tested positive for COVID-19, there was a significant greater burden on the women's physical health. Mental health and psychological status was similar in both groups. High quality of care during a pandemic is possible to deliver in a maternity setting, irrespective of the patient's COVID status.
\end{abstract}

\section{Title}

Health-related Quality of Life and Quality of Care in pregnant and postnatal women during the COVID-19 pandemic: a case control study

\section{Draft Authors}

Fátimah Alaya ${ }^{1,2}$

Amy P Worrall ${ }^{1}$

Fiona O'Toole ${ }^{1}$

Jillian Doyle ${ }^{1}$

Richard M Duffy ${ }^{1}$

Michael P Geary ${ }^{1,2}$

\section{Affiliations}


1. Rotunda Hospital, Parnell Street, Dublin 1, DO1 P5W9, Ireland

2. Royal College of Surgeons Ireland, St Stephen's Green, Dublin 2, D02 YN77, Ireland

\section{Editorial correspondence should be addressed to:}

Fatimah Alaya,

The Rotunda Hospital, Parnell Street, Dublin 1, DO1 P5W9, Ireland

fatimahalaya@rcsi.com

\section{Shortened Running Title}

HRQoL and QoC in perinatal women during COVID-19: a case control study

\section{Word Count}

2750

\section{Abstract \\ Objective}

To compare self-reported health related quality of life (HRQoL) and quality of care (QoC) received between pregnant and postnatal women during the COVID-19 pandemic at a tertiary unit.

\section{Design}

A prospective case-control study

\section{Population}

Pregnant women, [?]18 years of age, with capacity, who spoke English, and attended the tertiary unit during the pandemic.

\section{Methods}

18 perinatal women who tested positive for SARS-CoV-2 and 20 asymptomatic control perinatal women were recruited. Demographic characteristics were collected and all participants completed the Short Form (SF-12), Clinical Outcomes in Routine Evaluation-Outcome Measure (CORE-OM) and Quality from the Patient's Perspective (QPP). Means scores in both cohorts were compared.

\section{Main Outcome Measures}

Assessment of psychological distress, HRQoL, QoC and their correlates.

\section{Results}

$95 \%$ of the Non-COVID cohort were Caucasian, while $67 \%$ of the pregnant women who had COVID-19 were not Caucasian $\left(\mathrm{x}^{2}=16.01, \mathrm{p}<0.0001\right)$. The mean SF-12 for physical health and functionality in the COVID cohort had significantly lower scores (36.54 vs 49.21, 95\% CI [6.9 to 20.2], $\mathrm{p}<0.0002)$. There was no difference in mental health and wellbeing scores between the COVID and Non-COVID cohorts. The QoC experienced by both cohorts was similar and very positive.

\section{Conclusions}

Among pregnant women who tested positive for COVID-19, there was a significant greater burden on the women's physical health. Mental health and psychological status was similar in both groups. High quality of care during a pandemic is possible to deliver in a maternity setting, irrespective of the patient's COVID status.

\section{Abstract Word Count 241}

Funding 
The authors received no funding for this study and there are no disclosures of interest to declare

\section{Tweetable Abstract}

HRQoL and QoC in pregnant women during the COVID-19 pandemic: a case control

\section{Keywords}

Pregnancy, COVID-19, mental health, wellbeing, HRQoL, physical health, quality of care

\section{Introduction}

The Coronavirus Disease 2019 (COVID-19) pandemic caused by the severe acute respiratory syndrome coronavirus 2 (SARS-CoV-2) is a serious public health emergency. With rising numbers worldwide, and the threat of resurgence the impact of COVID-19 has been felt worldwide, in particular among vulnerable and high-risk cohorts such as pregnant women ${ }^{1,2}$. Immunosuppressed, pregnant, as well as Black, Asian and ethnic minority (BAME) cohorts are amongst the most vulnerable to the physical and psycho-social effects of COVID-191,3,4.

To, date the literature has reported no vertical transmission amongst pregnant women to the fetus in utero ${ }^{5-9}$. However, pregnant women in previous coronavirus epidemics of SARS-CoV and MERS-CoV often suffered more severe illness than their non-pregnant counterparts, with increased risk to mother and fetus ${ }^{10,11}$. The severity of COVID-19 in pregnant women is similar to that in non-pregnant adults ${ }^{12}$. There is also no evidence of increased risk of spontaneous abortion or preterm labour risk among pregnant women with COVID-19 12 . In fact, pregnant women are affected less by COVID-19 compared to the non-pregnant population ${ }^{13}$, though they may also be more at risk of severe illness if in respiratory failure ${ }^{7}$.

The COVID-19 pandemic and its sequelae have also caused mental health challenges and pregnant women and partners are no exception to this phenomenon ${ }^{14,15}$. Perinatal anxiety is an emerging consequence of the pandemic, and this anxiety can have implications for physical health too ${ }^{2,16,17}$. The impact of COVID-19 infection on pregnant women is both the direct impact of contracted COVID-19, but also the indirect impact of lockdown, social distancing and isolation measures, as part of the international efforts to stem the spread of the virus ${ }^{2}$.

Recommendations are that pregnant women with COVID-19 require specialist care in relation to diagnosis, management and prevention of complications for mother and baby ${ }^{13,18}$. Health-related quality of life (HRQoL) and the patient's perspective of the quality of care (QoC) received in healthcare settings are important outcome measures to assess for quality and impact of service delivery in maternity care. Quality of life and quality of care are commonly used measures to ascertain health-related quality of life (HRQoL) and the patient's perspective of the quality of care (QoC) received in healthcare settings. This survey evaluated HRQoL and QoC amongst pregnant and postpartum women in a tertiary unit in Dublin during the COVID19 outbreak. It was conducted to gain insight into the physical and mental wellbeing of pregnant women, and review the quality of care being provided during the COVID-19 pandemic. It aims to inform healthcare providers in providing pregnant women with the best care during this ongoing high-risk period.

\section{Methodology}

\section{Study design and participants}

This was a prospective case-control study on HRQoL and QoC for pregnant and postnatal women attending the Rotunda Hospital, a tertiary maternity hospital in Ireland, during the COVID-19 pandemic. Women were recruited from June to July 2020 to complete three validated questionnaires; The Short Form Health Survey (SF-12), CORE-Outcome Measure Questionnaire (CORE-OM) and Quality from the Patient's Perspective (QPP), as well as provide basic anonymous demographic information. Data was anonymously collected and was not linked to maternal medical records. All questionnaires were completed with recruited women by a single researcher for consistency. Women were recruited from prenatal, delivery and postnatal wards, as well as a voluntary COVID registry. The latter is an institutional registry of pregnant women who tested positive 
by polymerase chain reaction for SARS-CoV-2, and consented to being contacted for future research. 18 women who were SARS-CoV-2 positive consented to complete this research project. 20 perinatal women who had no signs, symptoms or positive SARS-CoV-2 results were recruited as case-controls. Women were eligible to participate if they attended the maternity unit during the COVID pandemic, if they had capacity, spoke English and were at least 18 years old.

\section{Questionnaire tools}

The Short Form Health Survey (SF-12), a widely used instrument for assessing general health and outcomes, was used to measure health related quality of life (HRQoL). The SF-12 measures physical and mental health using 12 questions. Summary scores for the physical component summary (PCS) and the mental component summary (MCS) were collated according to published guidelines ${ }^{19}$. A low score represents a poorer quality of physical or mental health.

The CORE-OM is a 34 question validated self-reported questionnaire, using a five-point scale that ranges from 'not at all' to 'most of/all the time'20. Four dimensions are captured, including: subjective wellbeing, problems and symptoms, life functioning and risk/harm. Mean and total scores were calculated as per developer recommendations to quantify the level of psychological global distress. A higher score represents a higher level of health and less global distress in the dimensions of wellbeing, problems and symptoms, and life functioning. A high score in the "risk/harm" domain represents a higher level of psychological distress.

The QPP is a 24-question model for assessing the patient's perception and experience of the quality of care in a healthcare environment ${ }^{21}$. The model consists of four dimensions: medical-technical competence of the healthcare provider, physical-technical conditions of the healthcare organization, degree of identityorientation in the attitudes and actions of the caregivers and the socio-cultural atmosphere of the healthcare organization. Questions were answered using a Likert scale (1-4) including: 'do not agree at all', 'slightly agree', 'mostly agree' and 'completely agree'.

\section{Statistical Analysis}

Data distribution was analysed by parametric and non-parametric tools using mean (standard deviation) or median (range), and student t-tests or Mann-Whitney tests respectively. Relations between nominal data was assessed by chi squared analysis. Pearson correlation was used for parametric correlation analysis. Significance was deemed a p value $<0.05$. Analysis was completed using Microsoft Office Excel Version 16 (2018) and GraphPad Prism software Version 8 (2020).

\section{Results \\ Demographics}

A total of 23 perinatal women who tested positive for SARS-CoV-2 consented to being contacted for further research. 18 of these were recruited to this study and completed the questionnaires, representing a $78 \%$ uptake rate. Of the 38 women who took part in the study, the mean age was $32(+-6.6), 66 \%$ were Caucasian, and $74 \%(\mathrm{n}=28)$ were multiparous women. Full demographics are detailed in Table 1. Age, parity, self-reported education level, marital status, income status, and general health and well-being were equally represented between COVID and Non-COVID cohorts. 95\% $(n=19)$ of the Non-COVID cohort were Caucasian, while $67 \%(n=12)$ of the pregnant women who had COVID-19 during pregnancy were not Caucasian. This relation between ethnicity and COVID status was significant $\left(\mathrm{x}^{2}=16.01, \mathrm{p}<0.0001\right)$.

Health, Wellbeing and Physical Questionnaire Results

Women's scores on the SF-12 domains and the CORE-OM are presented in Table 2. There was no difference in the overall SF-12 MCS between the two cohorts. Though the Non-COVID cohort had a slightly higher SF-12 score, it was not significant. In the SF-12 PCS domain for physical health and functionality the COVID cohort had significantly lower scores (36.54 vs 49.21, 95\% CI [6.9 to 20.2], p<0.0002) (Table 2). 
In sub-analyses of single items in the SF-12 questions women in the COVID cohort reported that having much less energy in comparison to women in the Non-COVID cohort (3.27 vs 1.8, 95\% CI [-2.362 to -0.5937], $\mathrm{p}<0.002)$. The COVID cohort also were much more limited when climbing stairs ( 0.083 vs $1.45,95 \%$ CI [0.132 to 1.1], $\mathrm{p}<0.015)$, felt significantly more pain interfering with normal work and life functioning $(2.28$ vs $1.1,95 \%$ CI [-2.12 to -0.24$], \mathrm{p}<0.016)$. The COVID cohort of women also reported significantly poorer results than the Non-COVID cohort when asked about feeling downhearted and blue $(2.55$ vs $3.56,95 \%$ CI [0.13 to 2.06], $\mathrm{p}<0.027)$.

There were no significant differences between cohorts in the overall CORE-OM score, or the CORE domains in wellbeing, functioning, problems/symptoms, or risk. However, in individual sub-analysis of questions the COVID cohort reports themselves as "lacking in energy and enthusiasm" more than the Non-COVID cohort ( 1 vs 2.11, 95\% CI [-1.93 to -0.29], $\mathrm{p}<0.009$ ), and reported suffering more from "aches, pains or other physical problems" (1.3 vs 2.5, 95\% CI [-2.16 to -0.24], $\mathrm{p}<0.016)$. Overall SF-12 MCS mental health and wellbeing question results in both groups correlated with CORE-OM results by Pearson analysis (-0.787, $95 \%$ CI [-0.9171 to -0.5067], $\mathrm{p}<0.0001)$.

Quality of Care Questionnaire Results

There were no significant differences in the overall scores between COVID and Non-COVID cohorts in relation to the quality of care received in their hospital interactions (Table 3). No differences were seen in the medical care received, the identity oriented approach of care, and the socio-cultural approaches to care. There was a statistically significant result between cohorts in the physical technical conditions domain, where the women in the COVID cohort reported significantly more satisfaction in relation to nutrition received, equipment provided in the room and hospital, and the quality of the hospital bed (4.11 vs 3.4, 95\% CI [-1.18 to -0.24], $\mathrm{p}<0.003)$.

\section{Discussion}

This cohort studied was reflective of the routine perinatal population that would attend our tertiary maternity unit normally. Notably, the proportion of non-Caucasian ethnic groups in the COVID-19 group was significantly higher. This reflects a national and international pattern whereby BAME populations, including the pregnant women within these cohorts, are seemingly more at risk of contracting COVID-194. The majority in both cohorts were in a partnership/co-habiting or married, had secondary or higher level of education, were coping or comfortable financially and reported a baseline good to very good level of health.

The impact of COVID-19 on anxiety and depression globally is a growing concern ${ }^{22}$. Pregnant women and their partners across the world have had to frequently adjust to the developing situation, with reports globally of increased anxiety in comparison to pre-pandemic levels ${ }^{17,23,24}$. Similarly, other reports have shown increased emotional eating among pregnant women during the pandemic ${ }^{25}$, and increased depression and anxiety in women and their husbands ${ }^{26}$. In assessing the HRQoL amongst women routinely attending for antenatal, delivery and postnatal maternity services, we found no significant difference between COVID and Non-COVID cohorts of women in relation to their mental health and psychological wellbeing. Both groups reported similar psychological wellbeing scores by two validated measures, SF-12 and CORE-OM, and those scores correlated closely $(-0.787,95 \%$ CI [-0.9171 to -0.5067$], \mathrm{p}<0.0001)$, reassuring their validity. A similar study in the UK was a pilot case-control comparison and reported no difference in anxiety between prenatal women with or without COVID-19 infection ${ }^{27}$. This study had smaller matched sample, and used different validated tools to assess anxiety and health. It found that rather than COVID-19 infection status, sociodemographic influences during the pandemic might have a greater impact on mental health among perinatal women ${ }^{27}$. Despite some literature reporting anxiety in perinatal women with COVID-19 during the pandemic, our data shows no different in the COVID and non-COVID-19 cohorts, which may suggest that the anxiety and mental health consequences seen during the COVID-19 pandemic are a product of the cultural and social environment rather than the SARS-CoV-2 infection itself.

Our data demonstrates there was a significantly higher physical burden for women who had COVID during the pandemic period. They reported higher levels of fatigue, pains, aches, shortness of breath and an 
impact of their ability to function day to day (Table 2). Many common gestational signs and symptoms of pregnancy such as physiological dyspnoea, altered pulmonary function, congestion, and fatigue are also the clinical manifestations of COVID-19 ${ }^{28-30}$, and we may have been underestimating the physical and physiological impact among the COVID cohort of women. It is unclear how the precise pathophysiology of COVID-19 in pregnancy can both be less severe generally amongst a pregnant cohort, but also be very severe among those women who have acute severe respiratory failure ${ }^{7,13,18}$. Some hypotheses include the protective hormonal environment attenuating severity during pregnancy, similar to hormonal effects shown in influenza infections among pregnant cohorts ${ }^{31}$. Another hypothesis is the combination of the immunological response to viral pathogens transitioning to a T-helper 2 milieu which favours anti-inflammatory cytokine expression and may reduce the severity of COVID-19 amongst pregnant women ${ }^{8}$. In spite of these hypotheses, much remains unknown, but our COVID cohort of pregnant women certainly felt an acute physical burden on their functional day-to-day capacity to be physically active.

The format of healthcare delivery in hospitals and healthcare settings has seen significant changes in an attempt to ameliorate the spread of COVID-19 and the risk to patients and healthcare workers. The healthcare environment has similarly adapted and re-developed to meet new emerging requirements to ensure safe and high quality care ${ }^{18}$. Hospitals worldwide, including our site, have prepared to face severe disruptions to routine protocols and procedures ${ }^{32}$. Similar to affected units globally, our site created a dedicated task force to ensure specific protocols were developed and applied across the tertiary unit; new ER triage protocols, patient isolation rooms, visitor restrictions, COVID-19 delivery and theatre practices were implemented as per the latest government guidance. Dedicated COVID-19 theatre measures were engaged and staff received up to date hospital developments as well as training in personal protective equipment, patient and self-management ${ }^{32,33}$. Collaboration and hospital multidisciplinary team work is notably at the core of successfully managing the pandemic and any subsequent resurgence ${ }^{32,34}$. The positive consequences of these proactive measures are clearly seen in the QoC results (Table 3). No differences were seen in the medical care received, the identity oriented approach of care, and the socio-cultural approaches to care in either COVID or non-COVID cohorts. The COVID cohort was significantly happier with the element of care received, specifically the physical technical domain, though both COVID and non-COVID groups had very positive results in this domain. The possible rationale for the COVID cohort expressing significantly more satisfaction in relation to nutrition received, equipment provided in the room and hospital, and the quality of the hospital bed (4.11 vs $3.4,95 \%$ CI [-1.18 to -0.24$], \mathrm{p}<0.003)$ possibly is as a result of the required isolation measures due to their COVID positive status. These women will have received single rooms, with en-suite bathrooms, better sleep, more focussed targeted postnatal care to minimise visits in and out of their rooms, and as such may have felt there was greater care, provision and quality given to them than they originally may have expected. The overall positive responses between both groups across a broad range of quality assessments from outpatient, inpatient, emergency room, medical and nursing care, to the quality of the hospital environment is very reassuring. Our results reinforce that a high level of care can be delivered and patient satisfaction can be maintained even while following stringent COVID-19 isolation and infection control management protocols.

The limitations of this study include the small sample number, the single centre studied, the bias associated with patient's self-selecting for future research, self-reporting results and variability between stages of pregnancy that each of the participants were at when surveyed. Similarly, the full maternal medical records were not accessed and as a result relevant history was excluded from the study. Future studies might look to power results with larger sample numbers, though post-hoc power analysis of our significant differences between cohorts was well powered, there is a risk of type two error in our results that were non-significant.

The management of COVID-19 in a pregnant women remains an evolving challenge for obstetricians and physicians. It is imperative that pregnant women receive the specialist holistic care during this time. There is real risk of increased maternal, fetal and pregnancy complications among labouring and pregnant women who lack support, and the literature supports proactively encouraging social connections during pregnancy ${ }^{35}$. Approaches used in previous worldwide crises and pandemics can be used, and specific strategies targeting maternal isolation or maternal stress such as psychological first aid and effective risk communication can 
reduce risk to women and their infants ${ }^{17,36}$. As the sequelae of the pandemic unfold, and as further surges arise, the care provided needs to focus on the added physical burden suffered by pregnant women who have contracted COVID-19, as well as psychological, emotional and mental health supports that too often are forgotten when other health crises present ${ }^{37,38}$. Additionally, maternity units must continue to meet the required demands for the expected quality of care, that we have shown is achievable, when caring for women, infants and their families during this ongoing pandemic.

\section{Conclusion}

In the cohort of perinatal women with COVID-19, there was a significantly greater burden on women's physical health and wellbeing, in particular there was reduced energy and motivation in women with SARSCoV-2. Mental health and psychological status was similar in both groups, this may reflect that the higher mental health needs are not linked to infection but rather the social, cultural and health care environment. High quality of care during a pandemic is possible to deliver in a maternity setting, irrespective of COVID status.

\section{Authorship}

FA collected the data. FA and AW analysed the data. FA, AW, FO'T, JD, RD and MG were involved in writing and reviewing the final version of the paper.

\section{Funding}

The authors received no funding for this study.

\section{Ethical Approval}

This study was given ethical approval by the Rotunda Hospital Research Ethics Committee on 23/06/2020, and research was conducted in line with local research policies, European GDPR guidelines, and the Helsinki Ethical Principles for Medical Research.

\section{Conflicts of Interest}

The authors have no conflicts of interest relevant to this publication to declare.

\section{References}

1. Dashraath P, Wong JLJ, Lim MXK, Lim LM, Li S, Biswas A, et al. Coronavirus disease 2019 (COVID-19) pandemic and pregnancy. Am J Obstet Gynecol. 2020;

2. Davenport MH, Meyer S, Meah VL, Strynadka MC, Khurana R. Moms Are Not OK: COVID-19 and Maternal Mental Health. Front Glob Women's Heal. 2020;

3. Iacobucci G. Covid-19: Admit ethnic minority pregnant women to hospital earlier, says NHS England. BMJ. 2020;

4. Knight M, Bunch K, Vousden N, Morris E, Simpson N, Gale C, et al. Characteristics and outcomes of pregnant women admitted to hospital with confirmed SARS-CoV-2 infection in UK: national population based cohort study. BMJ. 2020;

5. Chen Y, Peng H, Wang L, Zhao Y, Zeng L, Gao H, et al. Infants Born to Mothers With a New Coronavirus (COVID-19). Front Pediatr. 2020;

6. Li Y, Zhao R, Zheng S, Chen X, Wang J, Sheng X, et al. Lack of vertical transmission of severe acute respiratory syndrome Coronavirus 2, China. Emerg Infect Dis. 2020;

7. Karimi-Zarchi M, Neamatzadeh H, Dastgheib SA, Abbasi H, Mirjalili SR, Behforouz A, et al. Vertical Transmission of Coronavirus Disease 19 (COVID-19) from Infected Pregnant Mothers to Neonates: A Review. Fetal and Pediatric Pathology. 2020. 
8. Chen H, Guo J, Wang C, Luo F, Yu X, Zhang W, et al. Clinical characteristics and intrauterine vertical transmission potential of COVID-19 infection in nine pregnant women: a retrospective review of medical records. Lancet. 2020;

9. Chen S, Huang B, Luo DJ, Li X, Yang F, Zhao Y, et al. Clinical Features and placental pathological analysis of three cases of pregnant women with new coronavirus infection. Chinese J Pathol. 2020;

10. Wong SF, Chow KM, Leung TN, Ng WF, Ng TK, Shek CC, et al. Pregnancy and perinatal outcomes of women with severe acute respiratory syndrome. Am J Obstet Gynecol. 2004;

11. Alfaraj SH, Al-Tawfiq JA, Memish ZA. Middle East Respiratory Syndrome Coronavirus (MERS-CoV) infection during pregnancy: Report of two cases \& review of the literature. Journal of Microbiology, Immunology and Infection. 2019.

12. Yan J, Guo J, Fan C, Juan J, Yu X, Li J, et al. Coronavirus disease 2019 in pregnant women: a report based on 116 cases. Am J Obstet Gynecol. 2020;

13. Rasmussen SA, Jamieson DJ. Coronavirus Disease 2019 (COVID-19) and Pregnancy. Obstet Gynecol. 2020;

14. Bao Y, Sun Y, Meng S, Shi J, Lu L. 2019-nCoV epidemic: address mental health care to empower society. The Lancet. 2020.

15. Xu Z, Li S, Tian S, Li H, Kong L quan. Full spectrum of COVID-19 severity still being depicted. The Lancet. 2020.

16. Ceulemans M, Hompes T, Foulon V. Mental health status of pregnant and breastfeeding women during the COVID-19 pandemic: A call for action. Int J Gynecol Obstet. 2020;

17. Wu Y, Zhang C, Liu H, Duan C, Li C, Fan J, et al. Perinatal depressive and anxiety symptoms of pregnant women during the coronavirus disease 2019 outbreak in China. Am J Obstet Gynecol. 2020;

18. Jamieson DJ, Steinberg JP, Martinello RA, Perl TM, Rasmussen SA. Obstetricians on the Coronavirus Disease 2019 (COVID-19) Front Lines and the Confusing World of Personal Protective Equipment. Obstet Gynecol. 2020;

19. Ware JE, Kosinski M, Keller SD. A 12-Item Short-Form Health Survey: Construction of Scales and Preliminary Tests of Reliability and Validity. Med Care. 1996;

20. Skre I, Friborg O, Elgaroy S, Evans C, Myklebust LH, Lillevoll K, et al. The factor structure and psychometric properties of the Clinical Outcomes in Routine Evaluation - Outcome Measure (CORE-OM) in Norwegian clinical and non-clinical samples. BMC Psychiatry. 2013;

21. Larsson BW, Larsson G. Development of a short form of the Quality from the Patient's Perspective (QPP) questionnaire. Journal of Clinical Nursing. 2002.

22. Peteet JR. COVID-19 Anxiety. J Relig Health. 2020;

23. Lebel C, MacKinnon A, Bagshawe M, Tomfohr-Madsen L, Giesbrecht G. Elevated depression and anxiety symptoms among pregnant individuals during the COVID-19 pandemic. J Affect Disord. 2020;

24. Durankuş F, Aksu E. Effects of the COVID-19 pandemic on anxiety and depressive symptoms in pregnant women: a preliminary study. J Matern Neonatal Med. 2020;

25. Zhang J, Zhang Y, Huo S, Ma Y, Ke Y, Wang P, et al. Emotional Eating in Pregnant Women during the COVID-19 Pandemic and Its Association with Dietary Intake and Gestational Weight Gain. Nutrients. 2020 ;

26. Ahorsu DK, Imani V, Lin CY, Timpka T, Broström A, Updegraff JA, et al. Associations Between Fear of COVID-19, Mental Health, and Preventive Behaviours Across Pregnant Women and Husbands: An 
Actor-Partner Interdependence Modelling. Int J Ment Health Addict. 2020;

27. Kotabagi P, Fortune L, Essien S, Nauta M, Yoong W. Anxiety and depression levels among pregnant women with COVID-19. Acta Obstetricia et Gynecologica Scandinavica. 2020.

28. Nelson-Piercy C, Lowe S. Clinical practice guidelines in obstetric medicine. Obstetric Medicine. 2015.

29. Guan W, Ni Z, Hu Y, Liang W, Ou C, He J, et al. Clinical characteristics of coronavirus disease 2019 in China. N Engl J Med. 2020;

30. Mullins E, Evans D, Viner RM, O'Brien P, Morris E. Coronavirus in pregnancy and delivery: rapid review. Ultrasound in obstetrics \& gynecology : the official journal of the International Society of Ultrasound in Obstetrics and Gynecology. 2020.

31. Littauer EQ, Esser ES, Antao OQ, Vassilieva E V., Compans RW, Skountzou I. H1N1 influenza virus infection results in adverse pregnancy outcomes by disrupting tissue-specific hormonal regulation. PLoS Pathog. 2017;

32. Carenzo L, Costantini E, Greco M, Barra FL, Rendiniello V, Mainetti M, et al. Hospital surge capacity in a tertiary emergency referral centre during the COVID-19 outbreak in Italy. Anaesthesia. 2020.

33. Flemming S, Hankir M, Ernestus RI, Seyfried F, Germer CT, Meybohm P, et al. Surgery in times of COVID-19-recommendations for hospital and patient management. Langenbeck's Arch Surg. 2020;

34. Basile C, Combe C, Pizzarelli F, Covic A, Davenport A, Kanbay M, et al. Recommendations for the prevention, mitigation and containment of the emerging SARS-CoV-2 (COVID-19) pandemic in haemodialysis centres. Nephrol Dial Transplant. 2020;

35. Jago CA, Singh SS, Moretti F. Coronavirus Disease 2019 (COVID-19) and Pregnancy: Combating Isolation to Improve Outcomes. Obstet Gynecol. 2020;

36. Shah K, Kamrai D, Mekala H, Mann B, Desai K, Patel RS. Focus on Mental Health During the Coronavirus (COVID-19) Pandemic: Applying Learnings from the Past Outbreaks. Cureus. 2020;

37. MBRRACE-UK. Saving Lives, Improving Mothers' Care Rapid report: Learning from SARS-CoV-2related and associated maternal deaths in the UK. MBRRACE-UK. 2020.

38. Thapa SB, Mainali A, Schwank SE, Acharya G. Maternal mental health in the time of the COVID-19 pandemic. Acta Obstetricia et Gynecologica Scandinavica. 2020.

Table Reference List

Table 1. Patient demographics by COVID and Non-COVID cohort, with chi-squared analysis of relation, using a significance level of 0.05. Age was analysed by a comparison of the mean using t-test. Significance of $\mathrm{p}<0.0001, \mathrm{x}^{2}=16.01$ was seen between cohorts for ethnicity. Key: COVID - Coronavirus Disease 2019.

Table 2. Mean (+-SD) results for SF-12 Mental Health, SF-12 Physical Health, and CORE-OM Questionnaires in COVID and Non-COVID women. Analysis by comparison of means using unpaired t-tests. Key: COVID - Coronavirus Disease 2019, SF-12 Health Survey Short Form 12, CORE-OM , Core W - Wellbeing related questions, Core P - Problems and Symptoms related questions, Core F - Life Functioning questions, and Core R - Risk and Harm related questions.

Table 3. Mean (+-SD) results for QPP Questionnaire in COVID and Non-COVID women, analysis by parametric comparison of the means using unpaired t-tests. Scores range from 1 to 4 , with 4 (very good) being the highest rating for the quality of care received. QPP = Quality from the Patient's Perspective questionnaire.

Tables 
Table 1. Patient demographics by COVID and Non-COVID cohort, with chi-squared analysis of relation, using a significance level of 0.05. Age was analysed by a comparison of the mean using t-test. Significance of $\mathrm{p}<0.0001, \mathrm{x}^{2}=16.01$ was seen between cohorts for ethnicity. Key: COVID - Coronavirus Disease 2019.

\begin{tabular}{llll}
\hline Demographics & Non-COVID & COVID & \\
\hline Number (n) & 20 & 18 & \\
Age mean, (SD) & $32.6(7.6)$ & $31.3(5.542)$ & $\mathrm{p}=0.5$ \\
Ethnicity (n) Caucasian Other & 191 & 612 & $p<0.000$ \\
Parity (n) Primigravida Multiparous & 416 & 612 & $\mathrm{p}=0.3$ \\
Marital Status (n) Single Married Partnership/Co-habiting Divorced/Widowed & 17111 & 31140 & $\mathrm{p}=0.1$ \\
Education (n) None Secondary Level Higher Level & 0515 & 1314 & $\mathrm{p}=0.8$ \\
Income (n) Comfortable Coping Difficult & 1181 & 1080 & $\mathrm{p}=0.9$ \\
General Health (n) Very good Good Bad & 1550 & 1530 & $\mathrm{p}=0.5$ \\
\hline
\end{tabular}

Table 2. Mean $( \pm \mathrm{SD})$ results for SF-12 Mental Health, SF-12 Physical Health, and CORE-OM Questionnaires in COVID and Non-COVID women. Analysis by comparison of means using unpaired t-tests. Key: COVID - Coronavirus Disease 2019, SF-12 Health Survey Short Form 12, CORE-OM , Core W Wellbeing related questions, Core $\mathrm{P}$ - Problems and Symptoms related questions, Core F - Life Functioning questions, and Core R - Risk and Harm related questions.

\begin{tabular}{llll}
\hline Mental and Physical Wellbeing & Non-COVID & COVID & \\
\hline SF-12 MCS Mental mean, (SD) & $45.12(9.5)$ & $42.81(11.5)$ & $\mathrm{p}=0.432$ \\
SF-12 PCS Physical mean, (SD) & $49.21(7.2)$ & $35.64(12.6)$ & $\mathrm{p}<0.0002^{* *}$ \\
CORE W mean, (SD) & $1.08(0.90)$ & $1.15(0.84)$ & $\mathrm{p}=0.785$ \\
CORE P mean, (SD) & $0.76(0.69)$ & $1.14(0.79)$ & $\mathrm{p}=0.115$ \\
CORE F mean, (SD) & $0.52(0.42)$ & $0.73(0.60)$ & $\mathrm{p}=0.207$ \\
CORE R mean, (SD) & $0.09(0.28)$ & $0.02(0.08)$ & $\mathrm{p}=0.289$ \\
CORE-OM Total mean, (SD) & $0.76(0.52)$ & $0.61(0.48)$ & $\mathrm{p}=0.359$ \\
\hline
\end{tabular}

Table 3. Mean $( \pm \mathrm{SD})$ results for QPP Questionnaire in COVID and Non-COVID women, analysis by parametric comparison of the means using unpaired t-tests. Scores range from 1 to 4 , with 4 (very good) being the highest rating for the quality of care received. QPP = Quality from the Patient's Perspective questionnaire.

\begin{tabular}{llll}
\hline Quality from the Patient's Perspective (QPP) & Non-COVID & COVID & \\
\hline QPP Medical Technical Competence mean, (SD) & $3.63(0.52)$ & $3.72(0.56)$ & $\mathrm{p}=0.582$ \\
QPP Physical Technical Conditions mean, (SD) & $3.4(0.71)$ & $4.00(0.69)$ & $\mathrm{p}<0.0037^{* * *}$ \\
QPP Identity Oriented Approach mean, (SD) & $3.66(0.41)$ & $3.69(0.46)$ & $\mathrm{p}=0.846$ \\
QPP Socio Cultural Atmosphere mean, (SD) & $4.00(0.38)$ & $3.98(0.43)$ & $\mathrm{p}=0.806$ \\
QPP Total Patient Satisfaction Score mean, (SD) & $3.67(0.37)$ & $3.87(0.39)$ & $\mathrm{p}=0.804$ \\
\hline
\end{tabular}

